\title{
Capsule Commentary on Cheah et al., Ethnic Differences in Participation in Medical Check-ups Among the Elderly: Evidence from Malaysia
}

Yingzhe Yuan, $\mathrm{MPH}$

Department of Health Law, Policy and Management, Boston University School of Public Health, Boston, USA.

J Gen Intern Med 35(9):2834

DOI: $10.1007 / \mathrm{s} 11606-020-05794-2$

(c) Society of General Internal Medicine 2020

$\mathrm{T}$ he study by Cheah et al. ${ }^{1}$ examined differences in participation in medical check-ups between the ethnic groups among the elderly population in Malaysia. They also explored the associations between ethnicity and medical check-up participation in subgroups using multivariate analyses stratified by a series of demographic, insurance access, and health status variables. Controlling for demographic and health status variables, Chinese were 5.3\% more likely to participate in medical check-ups, compared to Malays. Stratifying the analysis by health status, the authors revealed that Chinese with diabetes, hypercholesterolemia, and hypertension had a higher medical check-up participation rate, compared to Malays with these health conditions. They suggested that Chinese elderly might have a high risk of suffering from the health conditions and the medical check-ups could be follow-up tests for them.

However, due to a limitation of cross-sectional data, it is possible that the medical check-ups played a role in the initial diagnoses of diabetes, hypercholesterolemia, and hypertension. Including health status obtained prior to and after the current study period could help address this limitation, if longitudinal data are available. To understand the ethnic difference in medical check-ups among elderly with health conditions, researchers should also look into a more complete set of patients' health status, their access to health care services, perception and knowledge of health, as well as other social, personal, and community factors, such as those listed in the Andersen's Behavioral Model. ${ }^{2}$

Published online March 30, 2020
For health policy makers and clinicians in Malaysia, the study provides evidence of an ethnic difference in participation in medical check-ups among the elderly and suggests them to target certain underserved subpopulations when allocating resources to improve the medical check-up rates. The study focuses on the topic of medical check-ups with the implication that efforts in preventative care are often effective and efficient in improving population health and maintaining health care costs. Furthermore, the findings of the study are generalizable to other countries with multiple ethnic groups, especially those sharing similar social and cultural characteristics with Malaysia.

Corresponding Author: Yingzhe Yuan, MPH; Department of Health Law, Policy and Management, Boston University School of Public Health, Boston, USA (e-mail: yuanyz@bu.edu).

Compliance with Ethical Standards:

Conflict of Interest: The author declares that she does not have a conflict of interest.

\section{REFERENCES}

1. Cheah YK, Meltzer D. Ethnic Differences in Participation in Medical Check-ups Among the Elderly: Evidence from Malaysia. J Gen Intern Med. (SPI 5766).

2. Andersen RM. Revisiting the behavioral model and access to medical care: does it matter?. Journal of health and social behavior. 1995 Mar 1:1-0.

Publisher's Note Springer Nature remains neutral with regard to jurisdictional claims in published maps and institutional affiliations. 